\title{
Variación en la distribución del manatí antillano (Trichechus manatus manatus) en la costa sur de Puerto Rico a través de censos aéreos en helicóptero
}

\section{Variation in the distribution of the Antillean manatee (Trichechus manatus manatus) on the southern coast of Puerto Rico through helicopter aerial surveys}

\author{
Antonio A. Mignucci-Giannoni ${ }^{1^{*}}$, Chabeli Iglesias-Escabi ${ }^{1,2}$, Raúl J. Rosario-Delestre $^{1}, y$ \\ Mayela Alsina-Guerrero ${ }^{1,3}$
}

\begin{abstract}
RESUMEN
El manatí antillano (Trichechus manatus manatus) está en peligro de extinción debido a actividades antrópicas, las cuales afectan tanto su salud como el uso de hábitats requeridos para su supervivencia. El presente estudio detalla la distribución espacial y uso de hábitat del manatí en la costa sur de Puerto Rico al hacer censos aéreos en helicóptero y relacionarlos con aspectos zoogeográficos, ambientales y oceanográficas. Se realizaron 33 censos aéreos en helicóptero, paralelos a la costa sur, desde el año 2001 al año 2015. Hubo un total de 488 avistamientos y 914 individuos, lo cual representa 13 manatíes por hora de esfuerzo y $12.4 \%$ de crías. No se encontró que factores de temporada, temperatura superficial del mar o las corrientes afecten su presencia. El uso de recursos de agua dulce y praderas de yerbas no presentó estadísticamente una relación, pero si es claro que son parte de su naturaleza y necesarios para su sobrevivencia. Sin embargo, la profundidad y el grado de protección que ofrecen las bahías si es determinante para predecir la presencia de manatíes. Un marcado incremento en el número de manatíes por hora de esfuerzo entre el año 2007 y año 2015 representa un posible aumento poblacional. Dado que la supervivencia de los manatíes en Puerto Rico está amenazada por diferentes factores naturales y antrópicos, este estudio, junto a los de telemetría y uso de hábitat, deben ayudar a designar en el futuro áreas protegidas críticas para su supervivencia.
\end{abstract}

Palabras claves: censos aéreos, helicóptero, Puerto Rico, Trichechus manatus, zoogeografía.

1 Centro de Conservación de Manatíes de Puerto Rico, Universidad Interamericana, PO Box 361715 San Juan, Puerto Rico 00936,mignucci@manatipr.org*

2 Facultad de Ciencias del Mar y Recursos Naturales, Universidad de Valparaíso. Av. Borgoño N. ${ }^{o} 16$ 344, Reñaca, Viña del Mar, Chile, chabeli.iglesias@yahoo.com

3 Georgia Aquarium, 225 Baker St. NW, Atlanta GA 30313 USA, malsina@georgiaaquarium.org Antonio A. Mignucci-Giannoni y Chabeli Iglesias-Escabí: estos autores contribuyeron igualmente en este trabajo. 


\begin{abstract}
The Antillean manatee (Trichechus manatus manatus) is endangered due to anthropogenic activities, which affect both the manatee's health and use of habitats required for their survival. The spatial distribution and use of the manatee habitat on the southern coast of Puerto Rico was obtained using helicopter aerial surveys and was related to zoogeographic, environmental and oceanographic factors. A total of 33 surveys were conducted along the southern coast from 2001 to 2015. There was a total of 488 sightings and 914 individuals, which represents 13 manatees per hour of effort, $12.4 \%$ being calves. No correlation was found between their presence and seasons, sea surface temperature, or currents. The use of freshwater and seagrass bed resources did not present a statistical relationship, but it is clear that they are part of their nature and necessary for their survival. However, the depth and degree of protection offered by the bays is decisive to predict the presence of manatees. A marked increase in the number of manatees per hour of effort between 2007 and 2015 represents a possible population growth. Given that the survival of manatees in Puerto Rico is threatened by different natural and anthropogenic factors, this study, together with studies on telemetry and habitat use, should help designate future protected areas critical for their survival.
\end{abstract}

Keywords: Aerial survey, helicopter, Puerto Rico, Trichechus manatus, zoogeography.

\section{INTRODUCCIÓN}

El manatí de las Indias Occidentales (Trichechus manatus) es una especie conformada por dos subespecies (Domning \& Hayek, 1986): el manatí de la Florida (T. manatus latirostris) y el manatí antillano ( $T$. manatus manatus). La distribución de esta especie se extiende desde la costa este de los Estados Unidos a partir de Georgia y Florida, por el Golfo de México y Mar Caribe, particularmente en las Antillas Mayores, hasta las costas de las Guyanas y Brasil en el Océano Atlántico occidental-ecuatorial (Deutsch et al. 2008). De estas áreas, Florida, Belice y Puerto Rico es donde se encuentran las poblaciones más estables (Self-Sullivan \& Mignucci-Giannoni, 2008; Self-Su1livan \& Mignucci-Giannoni, 2012).
Sin embargo, el manatí antillano es el mamífero marino en Puerto Rico con mayor peligro de extinción debido a actividades antrópicas, como la caza directa entre los siglos XVI y XX, choques con embarcaciones, invasión de hábitat y contaminación industrial (Mignucci-Giannoni et al. 2000; Mignucci, 2010; Bonde et al. 2012). Estas actividades perjudiciales afectan tanto la salud del manatí como el uso particular de hábitats específicos requeridos para su supervivencia. Además de las actividades antrópicas, se reportan como limitantes de su distribución en un área, la temperatura superficial del agua, la salinidad, la cantidad y el tipo de alimento disponible, bahías protegidas por la acción de las corrientes, el oleaje y los vientos, la profundidad de los cuerpos de 
agua y la claridad del agua (Lefebvre et al. 2000).

El uso de la costa sur de Puerto Rico por el manatí ha sido documentado desde los municipios: de Cabo Rojo hasta el de Patillas (Mignucci-Giannoni, 1989), y es en la costa sur, particularmente en los municipios de Peñuelas, Guayama y Salinas, donde se ha reportado una mayor incidencia de mortandad para la especie (Mignucci-Giannoni et al. 2000).

Un plan de recuperación para la población del manatí antillano en Puerto Rico fue publicado en 1986 (Rathbun \& Possardt, 1986). Desde que se listó el manatí como especie en peligro de extinción y la publicación de este plan de recuperación, el Servicio de Pesca y Vida Silvestre de los Estados Unidos y el Departamento de Recursos Naturales y Ambientales de Puerto Rico, han implementado varias actividades de recuperación, principalmente enfocadas en la educación ambiental, esfuerzos regulatorios, planes de monitoreo y uso de hábitat. Los censos aéreos llevados a cabo en varios intervalos desde la década de 1970 (Magor, 1979; Powell et al. 1981; Rathbun et al. 1985; Freeman \& Quintero, 1990; US Fish and Wildlife Service, datos no publicados) han ayudado a determinar los patrones de distribución, para así poder establecer áreas de protección del manatí. El establecimiento de un área de protección efectiva también requiere conocer los patrones de uso de recursos por parte del manatí, la relación entre estos y las amenazas actuales a esta especie y su hábitat.

Recursos potencialmente limitantes incluyen las praderas de yerbas marinas, la disponibilidad de agua dulce y protegidas (por ejemplo, bahías cerradas, poco profundas, protegidas del oleaje). La calidad de las praderas de yerbas marinas, el principal hábitat de alimentación de los manatíes de Puerto Rico (Mignucci-Giannoni \& Beck, 1998; Lefebvre et al. 2000), puede ser impactado por la sedimentación y otras fuentes de contaminación (Duarte et al. 2004). La calidad del agua también puede ser afectada por contaminación industrial y de agricultura (Drew et al. 2012).

El presente estudio detalla la distribución espacial y el uso de hábitat del manatí, particularmente en la costa sur de Puerto Rico, durante los últimos 15 años al utilizar censos aéreos en helicóptero, se investigó aspectos zoogeográficos en esta distribución y su asociación a variables ambientales y oceanográficas.

\section{MATERIALES Y MÉTODOS}

El estudio estuvo compuesto de una serie de censos aéreos paralelos a la costa sur de Puerto Rico, desde punta Águila, Cabo Rojo (17 $57.127^{\prime}$ N, $\left.67^{\circ} 12.880^{\prime} \mathrm{O}\right)$ hasta punta Yeguas, Maunabo (18 $0.729^{\prime} \mathrm{N}, 6^{\circ} 50.035^{\prime}$ O) (Fig. 1). 


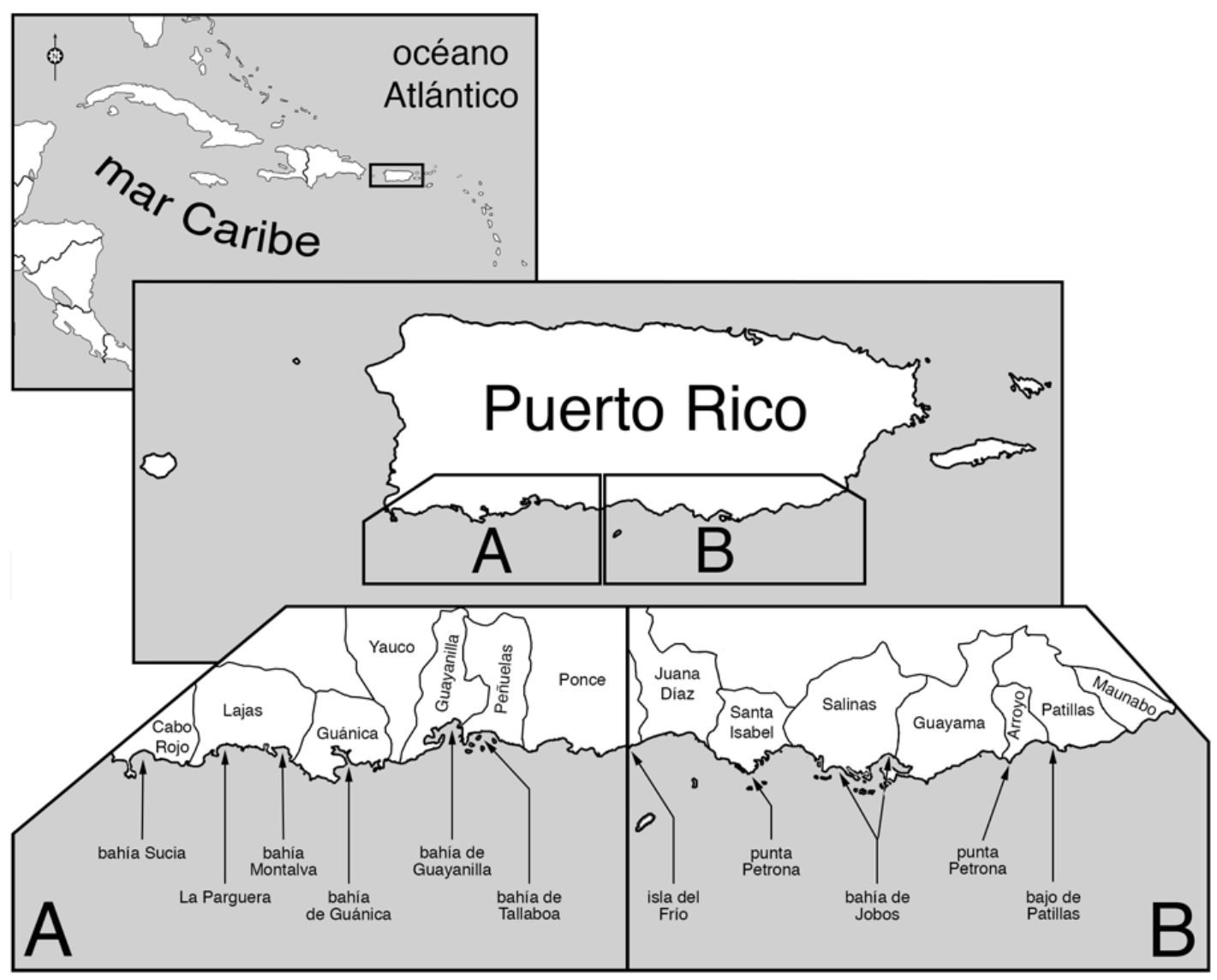

Fig. 1. Área de estudio en Puerto Rico, se detallan las dos secciones de la costa sur de la isla donde se llevaron a cabo los censos aéreos (A y B), y las localidades más relevantes

Fig. 1. Study area in Puerto Rico, detailing the two sections of the southern coast of the island where the aerial surveys were conducted (A and B), and the most relevant locations

Ya que los helicópteros han se escogió como plataforma usar probado ser útiles para obtener uno ligero, monomotor, Eurocopter datos valiosos sobre distribución y AS350B Écureuil (AStar) (Fig. 2). agrupaciones de manatíes (Provancha Dado la selección de su uso en vez de \& Provancha, 1988; Rathbun, 1988), avioneta, la metodología fue adaptada 


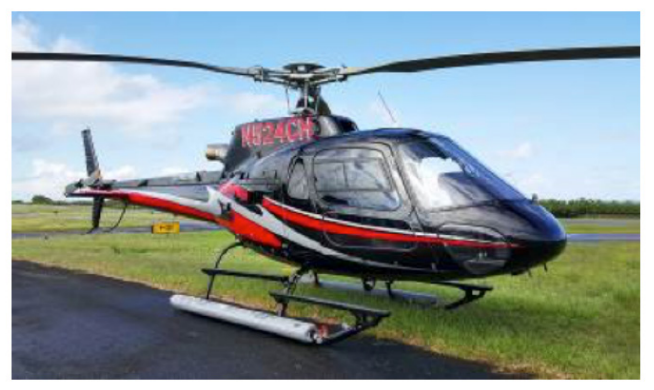

Fig. 2. Plataforma de avistamiento para los censos aéreos, un helicóptero ligero monomotor Eurocopter AS350B Écureuil (AStar) con flotadores de seguridad de despliegue automático en los patines de aterrizaje

Fig. 2. Sighting platform for aerial surveys, a Eurocopter AS350B Écureuil (AStar) single-engine light utility helicopter with automatic deployment safety floats on the landing skids

para helicópteros de los detallados en Packard et al. (1985), Packard (1985), Packard et al. (1986), Lefebvre \& Kochman (1991), Lefebvre et al. (1995), y Miller et al. (1998). Se utilizó el protocolo de seguridad para censos aéreos de Lefebvre (1995), que la tripulación llevara traje de aviación contra incendios (NOMEX®) III A FSL 27/P Flight Suit de Gibson \& Barnes, El Cajon, California), botas, chaleco salvavidas (HV-35 SPECOPS Special Operations Helicopter Vest de Switlik, Trenton, New Jersey) y botella de aire pequeña con regulador (HEED
3-HelicopterEmergency Egress Device 3 de Submersible Systems, Huntington Beach, California), y que el helicóptero llevara flotadores de seguridad de despliegue automático en los patines de aterrizaje (AerazurTm Emergency Flotation Gear de Zodiac Aerospace, Plaisir Cedex, Francia). Los censos aéreos se llevaron a cabo cuando las condiciones de vuelo fueron seguras y favorables, con muy pocas o nada de nubes y oleaje (Escala de Beaufort 0-3), en la mañana, durante aproximadamente 3.2 horas con una o dos paradas de 30-45 minutos para reabastecer combustible.

La costa sur de Puerto Rico (Fig. 1) se dividió en dos secciones como área de estudio para propósitos prácticos, suroeste (de Cabo Rojo a Ponce) y sureste (desde Juana Díaz a Maunabo). El plan de vuelo incluyó rutas predispuestas (Fig. 3), volando paralelo a la costa, a una distancia aproximadamente de $500 \mathrm{~m}$ de ella, a unos 150-185 m de altura y a una velocidad entre 165 y $200 \mathrm{~km} / \mathrm{h}$. Se tomaron datos de velocidad y altura cada 15 minutos. El esfuerzo se distribuyó uniformemente, al cubrir todas las áreas por igual y con el mismo tiempo. Para llevar a cabo censos sobre los pequeños cayos que quedan separados de la costa, se voló sobre la tierra de ellos con observadores que miraban hacia el agua a ambos lados del borde costero. 

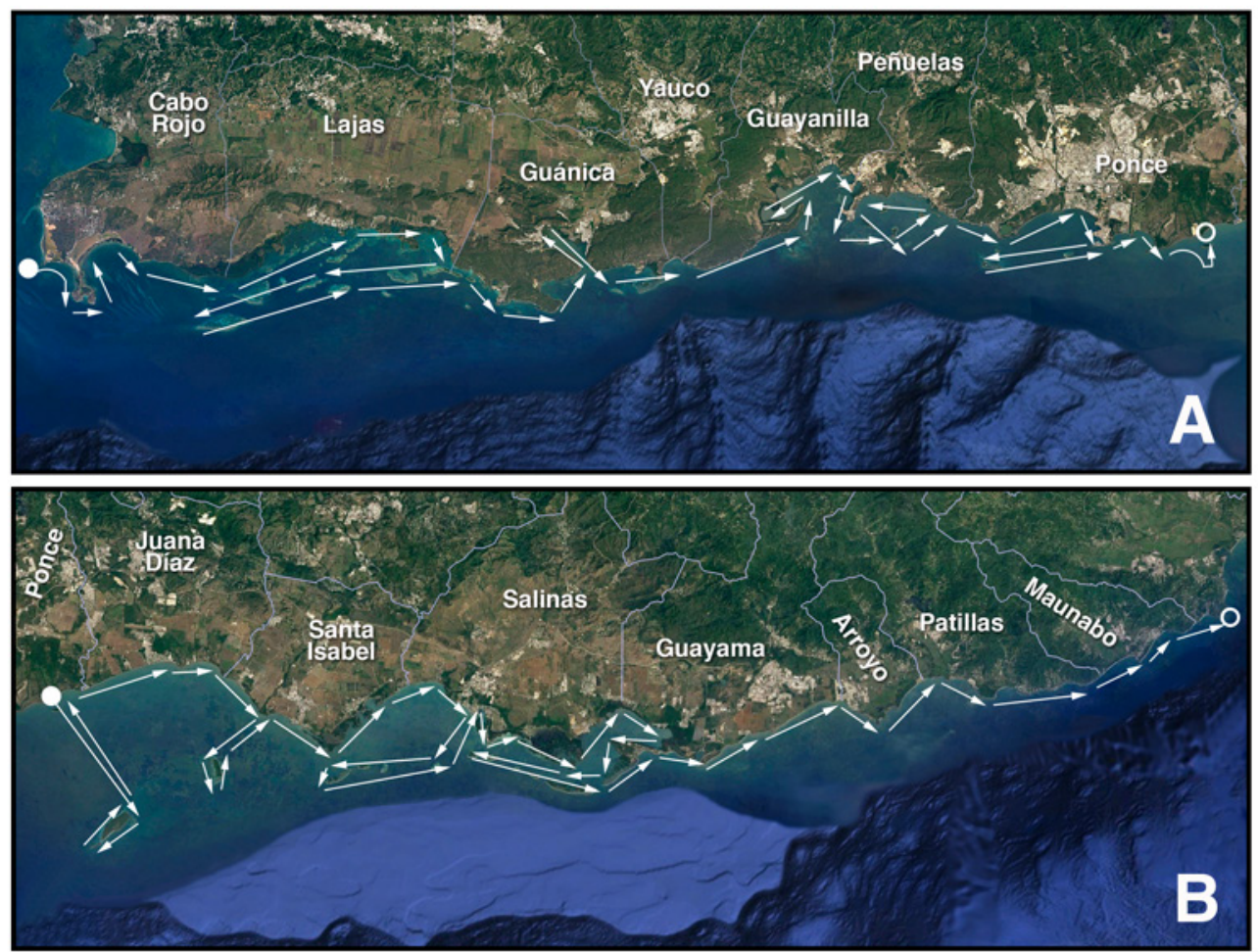

Fig. 3. Plan y transectos de vuelo para las costas suroeste (A) y sureste (B) de Puerto Rico. El círculo relleno, color blanco indica el comienzo del vuelo, el circulo vacío blanco indica el final del vuelo

Fig. 3. Flight plan and transects for the southwestern (A) and southeastern (B) coasts of Puerto Rico. The filled white circle is the beginning of the flight while the empty white circle is the end of the flight

En cada vuelo participó un piloto, tres observadores y una persona para tomar datos. Los tres tenían que llevar puestas gafas para el sol, polarizadas para eliminar el reflejo superficial del mar y el deslumbramiento, además audífonos acolchonados de cancelación de sonido (A20® Aviation Headset de Bose Corporation, Framing, Massachusetts), para la comunicación entre los tripulantes. Uno de los observadores iba sentado en el asiento del copiloto, mientras los otros dos iban sentados a cada lado del helicóptero; el registrador iba entre los dos observadores. Los tres buscaron en el agua principalmente en o cerca de la línea de transecto y periódicamente en el horizonte. Los avistamientos fueron escritos a mano de forma dual por el registrador en una ficha 
de datos y por el observador/copiloto en una carta náutica.

Cuando los animales fueron avistados durante las horas de esfuerzo, el helicóptero planeó estacionariamente o circuló al animal o grupo. Se estimó el número de manatíes adultos y el número de crías que había en cada grupo y se determinó el comportamiento.

Los comportamientos de grupo fueron clasificados en seis categorías según estudios anteriores: (1) alimentándose, (2) zambulléndose, (3) viajando, (4) descansando, (5) subiendo a la superficie y (6) apareándose. También, se tomó la hora en la cual se hizo el avistamiento, la localidad general, la latitud, la longitud y el observador/ copiloto marcó el avistamiento en la carta náutica.

Análisis de datos. Se organizaron los datos de la siguiente manera: número de adultos y de crías, comportamiento, clima, localidad general, latitud y longitud en tablas de Excel (Microsoft Excel, 2011). Los datos de avistamientos fueron ingresados también al programa Google Earth (2016) con su respectiva información. Al tener toda la información y adaptar la metodología utilizada en el trabajo de Drew et al. (2012), se buscó para cada avistamiento: A) la profundidad en metros utilizando cartas náuticas (North American Datum 1983); B) si se encontraba o no sobre praderas de yerbas marinas, para ello se utilizó los mapas del hábitat bentónico de la NOAA del año 2001 (Kendall et al. 2001); C) la distancia en metros a la fuente de agua dulce más cercana utilizando Google Earth, junto al mapa de los ríos principales de Puerto Rico (López-Marrero \& Villanueva-Colón, 2006); y D) el grado de protección de la bahía utilizando datos de batimetría y vientos para el Wave Exposure Model de la NOAA (WEMO 4.0; http://www.ccfhr.noaa.gov/stressors/wemo/).

Para comparar los avistamientos con las corrientes y la temperatura superficial del mar, se utilizaron unas gráficas de promedios de velocidad de estas, por cada estación del año del área del Caribe de la División de Oceanografía Física del Laboratorio de Oceanografía y Meteorología del Atlántico de la NOAA (http://www. aoml.noaa.gov/phod/index.php).

Se utilizaron estadísticas descriptivas y Chi-cuadrado para evaluar la presencia según la localidad en función de las variables de avistamiento sobre alimento disponible y grado de protección de la bahía. Como análisis post hoc se hizo un modelo paramétrico lineal general y un modelo no paramétrico, se utilizó el paquete np de R entre las variables ambientales: profundidad de los cuerpos de agua en los avistamientos, distancia a la fuente de agua dulce más cercana, alimento disponible $\mathrm{y}$ avistamientos de manatíes, por medio del software Rstudio (2016). 


\section{RESULTADOS}

Avistamientos y esfuerzo. Entre el año 2001 y año 2015 se realizaron 33 censos aéreos en helicóptero en la costa sur de Puerto Rico; 21 en el transecto suroeste de Cabo Rojo a Ponce, y 12 en el transecto sureste de Juana Díaz a Maunabo (Cuadro 1). La altura y la velocidad promedio de vuelo fueron $165.4 \mathrm{~m}(115.7-232.5 \mathrm{~m})$ y 155 kph (120-197.6 kph), respectivamente.

Se volaron un total de 105.7 horas, de las cuales 70.5 fueron de esfuerzo en avistar manatíes. Hubo un total de 488 avistamientos y 914 individuos (Cuadro 2), lo cual representa 6.9 avistamientos y 13.0 individuos de manatí por hora de esfuerzo (Cuadro 2). El número de manatíes aumentó desde el año 2001 y el año 2002 con números iniciales de alrededor de 5-7 animales, a más de 17 manatíes por hora de esfuerzo a partir del año 2007 (Cuadro 2, Fig. 4).

Cuadro 1. Censos aéreos de manatíes en la costa sur de Puerto Rico desde el 2001 al 2015

Table 1. Aerial surveys of manatees along the southern coast of Puerto Rico from 2001 to 2015

\begin{tabular}{|c|c|c|c|c|c|c|c|c|}
\hline Fecha & $\begin{array}{l}\text { Horas } \\
\text { de vuelo }\end{array}$ & $\begin{array}{l}\text { Horas } \\
\text { de es- } \\
\text { fuerzo }\end{array}$ & $\begin{array}{l}\text { Promedio } \\
\text { altura }(m)\end{array}$ & $\begin{array}{l}\text { Promedio } r \\
(k p h)\end{array}$ & $\begin{array}{l}\text { Avista- } \\
\text { mientos }\end{array}$ & Individuos & $\begin{array}{l}\text { Parejas } \\
\text { de ma- } \\
\text { dre/cría }\end{array}$ & $\begin{array}{l}\text { Promedio } \\
\text { de grupo }\end{array}$ \\
\hline \multicolumn{9}{|c|}{ Transecto suroeste } \\
\hline 17 ene 2001 & 3.8 & 2.7 & 171.4 & 166.7 & 13 & 25 & 5 & 1.9 \\
\hline 04 abr 2001 & 3.5 & 2.6 & 212.6 & 137.4 & 11 & 15 & 2 & 1.4 \\
\hline 18 jul /2001 & 4.2 & 3.2 & 161.5 & 125.9 & 4 & 9 & 0 & 2.3 \\
\hline 21 nov 2001 & 2.9 & 2.1 & 148.0 & 172.1 & 15 & 29 & 8 & 1.9 \\
\hline 19 abr 2002 & 2.8 & 1.9 & 141.9 & 155.4 & 7 & 14 & 3 & 2.0 \\
\hline 19 jul 2002 & 2.5 & 1.7 & 185.3 & 155.6 & 3 & 5 & 1 & 1.7 \\
\hline 20 sep 2002 & 3.4 & 2.5 & 142.2 & 197.6 & 17 & 28 & 4 & 1.6 \\
\hline $06 \operatorname{dic} 2002$ & 3.2 & 2.3 & 163.9 & 149.3 & 10 & 13 & 0 & 1.3 \\
\hline 17 feb 2003 & 2.8 & 1.8 & 185.9 & 179.6 & 5 & 9 & 1 & 1.8 \\
\hline 28 abr 2003 & 2.8 & 2.0 & 115.7 & 170.4 & 9 & 11 & 1 & 1.2 \\
\hline 13 feb 2004 & 4.5 & 1.6 & 169.8 & 187.8 & 6 & 15 & 1 & 2.5 \\
\hline 20 ene 2005 & 3.5 & 1.7 & 152.4 & 161.3 & 12 & 20 & 4 & 1.7 \\
\hline 04 jun 2005 & 2.6 & 1.6 & 166.8 & 167.1 & 8 & 10 & 1 & 1.3 \\
\hline 09 dic 2005 & 2.8 & 2.2 & 152.4 & 159.8 & 8 & 15 & 2 & 1.9 \\
\hline
\end{tabular}




\begin{tabular}{|c|c|c|c|c|c|c|c|c|}
\hline Fecha & $\begin{array}{l}\text { Horas } \\
\text { de vuelo }\end{array}$ & $\begin{array}{l}\text { Horas } \\
\text { de es- } \\
\text { fuerzo }\end{array}$ & $\begin{array}{l}\text { Promedio } \\
\text { altura }(m)\end{array}$ & $\begin{array}{l}\text { Promedio v } \\
(k p h)\end{array}$ & $\begin{array}{l}\text { Avista- } \\
\text { mientos }\end{array}$ & Individuos & $\begin{array}{l}\text { Parejas } \\
\text { de ma- } \\
\text { dre/cría }\end{array}$ & $\begin{array}{l}\text { Promedio } \\
\text { de grupo }\end{array}$ \\
\hline 11 sep 2007 & 3.3 & 2.0 & 152.4 & 161.7 & 16 & 39 & 3 & 2.4 \\
\hline 14 may 2008 & 3.3 & 1.8 & 149.3 & 157.4 & 13 & 35 & 7 & 2.7 \\
\hline 26 nov 2008 & 2.9 & 1.9 & 158.5 & 157.4 & 22 & 37 & 5 & 1.7 \\
\hline 29 ene 2010 & 3.1 & 1.9 & 142.2 & 146.7 & 17 & 22 & 1 & 1.3 \\
\hline 22 oct 2010 & 3.2 & 1.9 & 160.0 & 143.5 & 20 & 42 & 6 & 2.1 \\
\hline 13 feb 2012 & 3.0 & 1.8 & 152.4 & 157.4 & 33 & 69 & 8 & 2.1 \\
\hline 11 jun 2015 & 3.7 & 2.6 & 232.5 & 120.0 & 27 & 55 & 3 & 2.0 \\
\hline Total & 67.6 & 43.6 & & & 276 & 517 & 66 & \\
\hline Promedio & $3.2 \pm 0.5$ & $2.1 \pm 0.4$ & $162.7 \pm 25.4$ & $158.6 \pm 18.4$ & $13.1 \pm 7.7$ & $24.6 \pm 16.6$ & $3.1 \pm 2.5$ & $1.9 \pm 1.5$ \\
\hline Mín./ Máx. & $2.5 / 4.5$ & $1.6 / 32$ & $115.7 / 235.5$ & $120.0 / 197.6$ & $3 / 33$ & $5 / 69$ & $0 / 8$ & $1 / 11$ \\
\hline \multicolumn{9}{|c|}{ Transecto sureste } \\
\hline 28 jun 2002 & 3.4 & 2.4 & 165.8 & 124.1 & 6 & 11 & 2 & 1.8 \\
\hline 13 sep 2002 & 3.2 & 2.3 & 180.8 & 141.9 & 13 & 28 & 4 & 2.2 \\
\hline 13 dic 2002 & 3.5 & 2.5 & 193.8 & 142.8 & 9 & 16 & 0 & 1.8 \\
\hline 07 feb 2003 & 2.8 & 2.0 & 179.8 & 148.2 & 6 & 18 & 4 & 3.0 \\
\hline $24 \operatorname{mar} 2003$ & 3.2 & 2.3 & 184.4 & 170.0 & 23 & 40 & 6 & 1.7 \\
\hline 20 sep 2003 & 3.5 & 2.2 & 175.8 & 150.0 & 12 & 18 & 2 & 1.5 \\
\hline 23 ene 2004 & 3.8 & 3.2 & 176.1 & 152.0 & 30 & 56 & 5 & 1.9 \\
\hline 01 abr 2004 & 3.7 & 2.4 & 198.1 & 173.7 & 12 & 30 & 1 & 2.5 \\
\hline 21 ene 2005 & 2.5 & 1.8 & 152.4 & 140.8 & 28 & 55 & 9 & 2.0 \\
\hline 01 jun 2005 & 2.1 & 1.4 & 146.8 & 172.9 & 15 & 26 & 1 & 1.7 \\
\hline $01 \operatorname{mar} 2012$ & 4.1 & 2.7 & 155.8 & 126.6 & 36 & 65 & 6 & 1.8 \\
\hline 12 jun 2015 & 2.4 & 1.9 & 130.2 & 143.5 & 22 & 34 & 7 & 1.5 \\
\hline Total & 38.0 & 26.9 & & & 212 & 397 & 47 & \\
\hline Promedio & $3.2 \pm 0.6$ & $2.2 \pm 0.4$ & $170.0 \pm 20.2$ & $148.9 \pm 16.4$ & $17.7 \pm 9.9$ & $33.1 \pm 17.6$ & $3.9 \pm 2.8$ & $1.9 \pm 1.5$ \\
\hline Mín./ Máx. & $2.1 / 4.1$ & $1.4 / 3.2$ & $130.2 / 198.1$ & $124.1 / 173.7$ & $6 / 36$ & $11 / 65$ & $0 / 9$ & $1 / 12$ \\
\hline \multicolumn{9}{|c|}{ Costa sur de Puerto Rico } \\
\hline Total & 105.7 & 70.5 & & & 488 & 914 & 113 & \\
\hline Promedio & $3.2 \pm 0.5$ & $2.1 \pm 0.4$ & $165.4 \pm 23.6$ & $155.0 \pm 18.1$ & $14.8 \pm 8.7$ & $27.7 \pm 17.2$ & $3.4 \pm 2.6$ & $1.9 \pm 1.5$ \\
\hline Mín./ Máx. & $2.1 / 4.5$ & $1.4 / 3.2$ & $115.7 / 232.5$ & $120.0 / 197.6$ & $3 / 36$ & $5 / 69$ & $0 / 9$ & $1 / 12$ \\
\hline
\end{tabular}




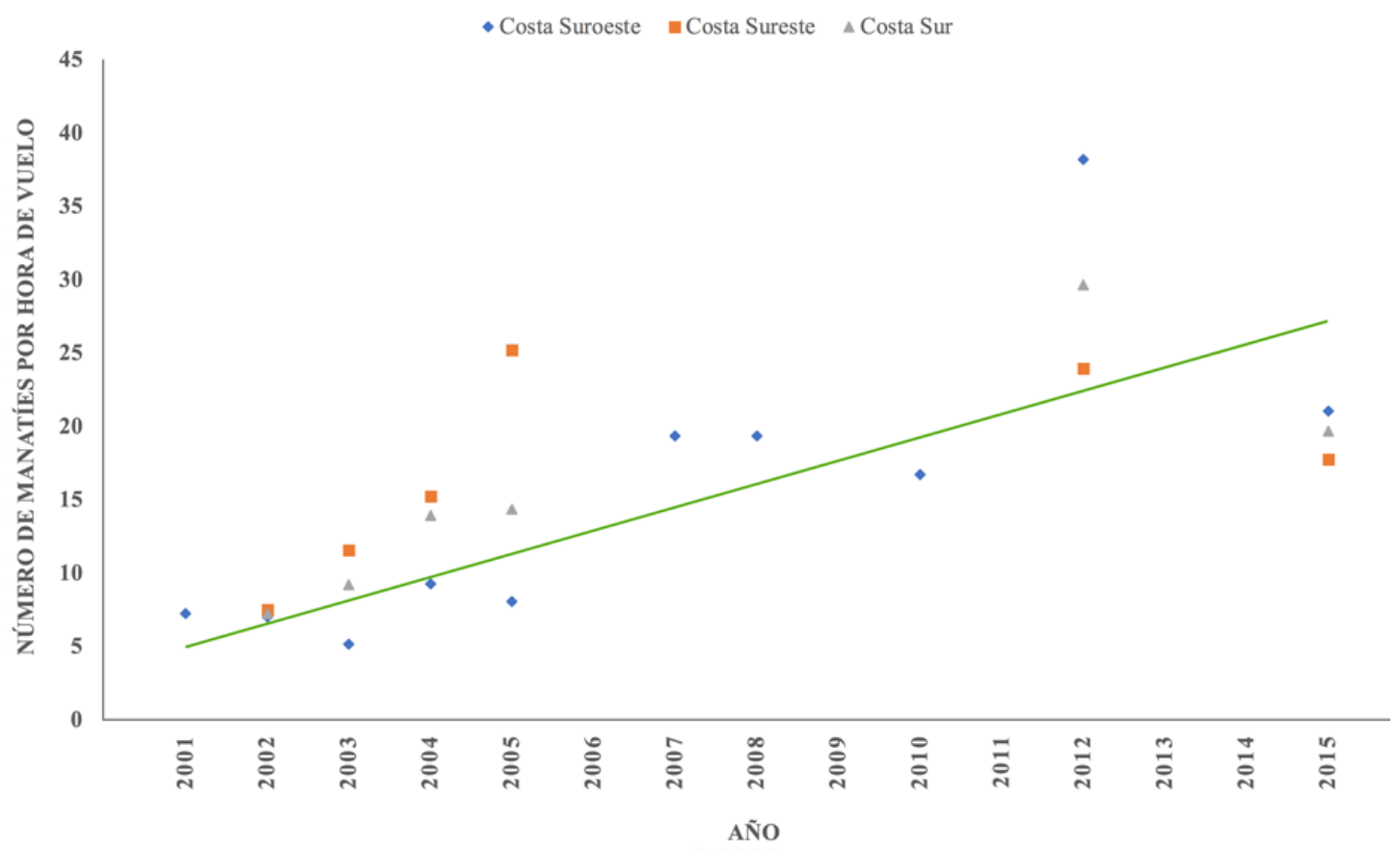

Fig. 4. Número de manatíes por hora de esfuerzo desde el 2001 al 2015, por costa de Puerto Rico, se demuestra la pendiente positiva indicativa de un posible aumento poblacional. Los diamantes azules representan la costa suroeste, los cuadrados anaranjados representa la costa sureste, y los triángulos grises representa la costa sur completa

Fig. 4. Number of manatees per hour of effort from 2001 to 2015 in each coast of Puerto Rico, demonstrating the positive slope indicative of a possible population increase. Blue diamonds represent the southwestern coast, orange squares represent the southeastern coast, and gray triangles represent the entire southern coast

Agrupaciones. Los manatíes (hasta doce), se encontraron princifueron encontrados en agrupaciones palmente alimentándose juntos o en pequeñas de uno o dos animales $(\mathrm{n}=$ comportamientos de reproducción. De 488, promedio 1.9, mínimo 1, máximo todos los avistamientos, $113(23.2 \%)$ 12, SD 1.5, Cuadro 1, Fig. 5). Agru- fueron de madres con crías, para un paciones mayores a cuatro animales $12.4 \%$ crías avistadas (Cuadro 2). 

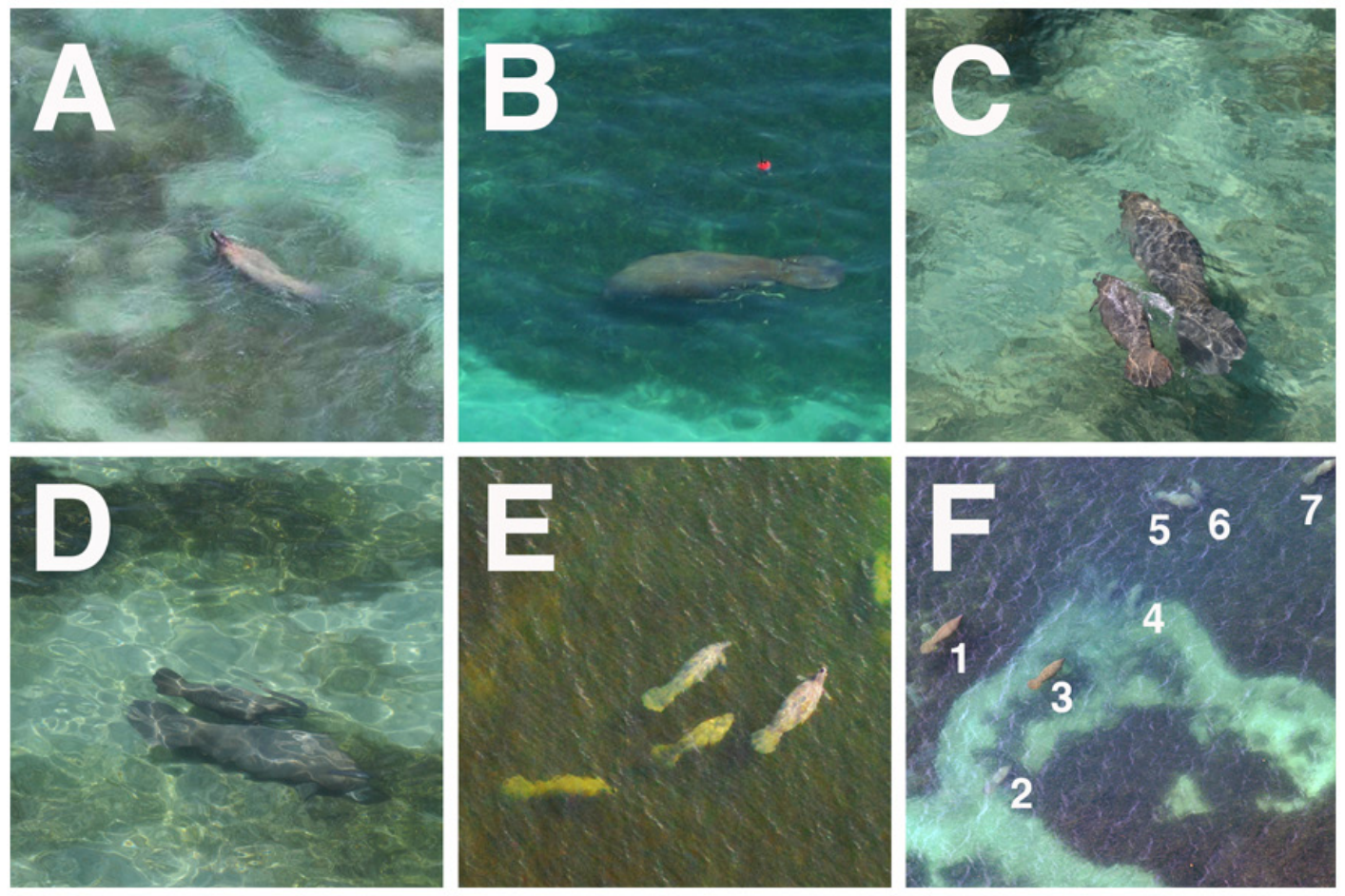

Fig. 5. Fotos aéreas de manatíes antillanos durante los censos de helicóptero en la costa sur de Puerto Rico, detalla diferentes agrupaciones: A) animal solitario, B) animal con transmisor de satélite alimentándose en una pradera de yerba marina, C) pareja de madre/cría, D) pareja de madre/cría alimentándose en parcho de yerbas marinas, E) cuatro manatíes juntos sobre pradera de yerba marina, F) siete manatíes en una agregación ligera, incluye una madre con cría (números 5 y 6 ) sobre parcho de arena y pradera de yerba marina

Fig. 5. Aerial photos of Antillean manatees during the helicopter aerial surveys on the southern coast of Puerto Rico, detailing different groupings: A) single animal, B) animal with satellite transmitter feeding on a seagrass bed, C) mother/ calf pair, D) mother/calf pair feeding on seagrass bed, E) four manatees together on a seagrass bed, F) seven manatees on a light aggregation, including a mother with calf (numbers 5 and 6) on a sand patch and seagrass bed 
Cuadro 2. Avistamientos de manatíes por costa y correlación con esfuerzo

Table 2. Manatee sightings by coast and correlation with effort

\begin{tabular}{llll}
\hline & $\begin{array}{l}\text { Costa } \\
\text { suroeste }\end{array}$ & $\begin{array}{l}\text { Costa } \\
\text { sureste }\end{array}$ & $\begin{array}{l}\text { Costa } \\
\text { sur }\end{array}$ \\
\hline Número de avistamientos & 276 & 212 & 488 \\
Número de manatíes & 517 & 397 & 914 \\
Número de parejas madre/cría & 66 & 47 & 113 \\
Horas de esfuerzo & 43.6 & 26.9 & 70.5 \\
Número de avistamientos por hora de esfuerzo & 6.3 & 7.9 & 6.9 \\
Número de manatíes por hora de esfuerzo & 11.9 & 14.8 & 13.0 \\
Número de parejas madre/cría por hora de esfuerzo & 1.5 & 1.7 & 1.6 \\
Número de manatíes por hora de esfuerzo 2001 & 7.4 & - & - \\
Número de manatíes por hora de esfuerzo 2002 & 7.1 & 7.6 & 7.4 \\
Número de manatíes por hora de esfuerzo 2003 & 5.3 & 11.7 & 9.3 \\
Número de manatíes por hora de esfuerzo 2004 & 9.4 & 15.4 & 14.0 \\
Número de manatíes por hora de esfuerzo 2005 & 8.2 & 25.3 & 14.5 \\
Número de manatíes por hora de esfuerzo 2007 & 19.5 & - & - \\
Número de manatíes por hora de esfuerzo 2008 & 19.5 & - & - \\
Número de manatíes por hora de esfuerzo 2010 & 16.8 & - & - \\
Número de manatíes por hora de esfuerzo 2012 & 38.3 & 24.1 & 29.8 \\
Número de manatíes por hora de esfuerzo 2015 & 21.2 & 17.9 & 19.8 \\
Porciento de parejas de madre/cría por & $23.9 \%$ & $22.2 \%$ & $23.2 \%$ \\
avistamientos & & & \\
Porciento de parejas de madre/cría por individuos & $12.7 \%$ & $11.8 \%$ & $12.4 \%$ \\
\hline
\end{tabular}

Comportamiento. Los manatíes fueron avistados mayormente viajando $(26.3 \%)$ o en actividad de reproducción $(24.7 \%)$. En menor grado, $17.7 \%$ de los manatíes fueron avistados alimentándose, $15.5 \%$ de ellos subiendo a la superficie para respirar, $10.8 \%$ estaban descansando, y $5.1 \%$ de ellos zambulléndose.

Distribución temporal. Los censos aéreos cubrieron las cuatro estaciones templadas del año: primavera (marzo-mayo, 21.2\%), verano (junio-agosto $24.2 \%$ ), otoño (septiembre-noviembre, $21.2 \%$ ) e invierno (diciembre-febrero, 33.4\%), al igual que las dos estaciones ecuatoriales del año: temporada seca (diciembre-mayo, $50 \%$ ) y de lluvia (junio-noviembre, $50 \%$ ). Sin embargo, la distribución de avistamientos de individuos y grupos madre/cría no fueron uniformes. La 
temporada seca vis a vis temporada lluviosa, obtuvo un porcentaje mayor en cada uno de estos tres renglones (avistamientos $56.6 \%$, individuos avistados $57.6 \%$, y grupos madre/cría 56.6\%). De forma similar, un porcentaje mayor fue obtenido en los tres renglones para las temporadas de primavera $(31.6 \%$, $32.8 \%$ y $34.4 \%$, respectivamente) y otoño $(32.3 \%$, 32.4\% y $36.7 \%$, respectivamente). Marcadamente, los meses de invierno resultaron en el menor porcentaje de avistamientos, individuos y grupos madre/cría.
Distribución espacial. Los manatíes fueron avistados en todos los municipios de la costa sur de Puerto Rico, menos Yauco y Maunabo (Cuadro 3, Figs. 6 y 7). Sin embargo, la distribución no fue uniforme, tanto en número de avistamientos, individuos, parejas madre/cría o áreas de reproducción. Los municipios con mayor número de estos avistamientos e individuos fueron Salinas $(18.0 \%$ y $18.9 \%$, respectivamente), y Guánica (16.6\% y 18.2\%, respectivamente). En comparación, los municipios con mayor número de parejas madre/cría fueron Salinas (18.6\%),

Cuadro 3. Relación de avistamientos de manatíes en los municipios costeros del sur de Puerto Rico

Table 3. List of manatee sightings by coastal municipalities of southern Puerto Rico

\begin{tabular}{lcrrr}
\hline Municipio & $\begin{array}{c}\text { Porciento } \\
\text { avistamientos }\end{array}$ & $\begin{array}{c}\text { Porciento } \\
\text { individuos }\end{array}$ & $\begin{array}{l}\text { Porciento } \\
\text { madre/cría }\end{array}$ & $\begin{array}{c}\text { Promedio de } \\
\text { agrupación }\end{array}$ \\
\hline Cabo Rojo & 7.0 & 6.1 & 9.7 & 1.6 \\
Lajas & 9.2 & 8.8 & 15.0 & 1.8 \\
Guánica & 16.6 & 18.2 & 15.9 & 2.0 \\
Yauco & 0 & 0 & 0 & - \\
Guayanilla & 9.0 & 8.0 & 5.3 & 1.7 \\
Peñuelas & 8.8 & 9.7 & 7.1 & 2.1 \\
Ponce & 6.1 & 5.9 & 5.3 & 1.8 \\
Juana Díaz & 1.6 & 1.9 & 0 & 2.1 \\
Santa Isabel & 8.0 & 7.7 & 5.3 & 1.8 \\
Salinas & 18.0 & 18.9 & 18.6 & 2.0 \\
Guayama & 8.8 & 9.1 & 8.0 & 1.9 \\
Arroyo & 2.9 & 2.2 & 3.5 & 1.4 \\
Patillas & 3.9 & 3.6 & 6.2 & 1.7 \\
Maunabo & 0 & 0 & 0 & - \\
Total & 100.0 & 100.0 & 100.0 & 1.9 \\
\hline
\end{tabular}


Guánica (15.9\%) y Lajas (15.0\%), lo cual difirió de las áreas de actividad reproductiva en las que en Guánica (30.4\%), Guayama (13.0\%), Juana Díaz (13.0\%) y Peñuelas (13.0\%) fueron más frecuentes. De particular interés, se documentó el uso por parte de manatíes de los cayos lejanos a la costa en La Parguera (arrecife Margarita, El Palo, Media Luna y Turrumote), Tallaboa (cayo María Langa, cayo Palomas, cayo Río y cayo Caribe), Ponce (cayo Cardona e isla del Frío), Santa Isabel (cayo Barbería), la cayería del estuario de la bahía de Jobos en Salinas y Guayama, y el uso repetido de la bahía Bioluminiscente en la Parguera. Sin embargo, no se observaron manatíes durante el estudio en la isla de Caja de Muerto al sur de Ponce y Juana Díaz.

Zoogeográficamente, se evaluó la relación entre avistamientos y (1) si este se encontraba, o no, sobre praderas de yerbas marinas, (2) profundidad, (3) distancia a la fuente de agua dulce más cercana, (4) temperatura superficial del mar, (5) grado de protección que proveen las bahías de los vientos, y (6) velocidad de las corrientes. Una leve mayoría $(52.1 \%)$ de los animales se encontraron sobre praderas de yerbas marinas, mientras que para parejas madre/cría no se encontraron diferencias significativas $(P=0.2103$ para dentro de praderas y $P=0.2236$ para afuera de praderas).
La distancia promedio desde los avistamientos de manatíes a la fuente de agua dulce (río o quebrada) más cercana en la costa sur fue de $6.8 \mathrm{~km}$ ( $\mathrm{n}=488$, mínimo $0 \mathrm{~km}$, máximo 35.4 $\mathrm{km}$, SD $8.9 \mathrm{~km}$ ). Distancia promedio entre los avistamientos de parejas de madre/cría a la fuente de agua dulce más cercana fue de $8.5 \mathrm{~km}(\mathrm{n}=121$, mínimo $0 \mathrm{~km}$, máximo $35.4 \mathrm{~km}$, SD $10.2 \mathrm{~km})$, pero estadísticamente esto no fue significativo $(P=0.0784)$. Se documentó la entrada de manatíes a dos ríos en la costa sur de Puerto Rico, el río Loco de Guánica y el río Bucaná en Ponce.

No se encontró relación entre la distribución temporal o espacial de los manatíes en la costa sur de Puerto Rico y la temperatura superficial del $\operatorname{mar}(P=0.2367)$, al tomar en cuenta que en promedio el grado de diferencia entre el agua más fría y más cálida es de $2^{\circ} \mathrm{C}\left(27^{\circ} \mathrm{C}\right.$ entre enero y marzo, y $29^{\circ} \mathrm{C}$ entre julio y septiembre) y el límite inferior observado con efectos adversos clínicos para la especie en áreas templadas (i. e., Florida) es menor a los $22^{\circ} \mathrm{C}$ (Reep \& Bonde, 2010). Tampoco, hubo una relación significativa entre la distribución temporal o espacial de los manatíes en la costa sur y la velocidad de las corrientes $(P=0.2769)$. La profundidad promedio para los avistamientos, tanto en general como para parejas de madre/cría, fue de $3.4 \mathrm{~m}$ (n =488, mínimo $0.3 \mathrm{~m}$, máximo $19.5 \mathrm{~m}$, SD $2.9 \mathrm{~m}$ ), y se encontró que esto es 
Cuadro 4. Relación entre porcientos de avistamientos de manatíes en la costa sur de Puerto Rico vs. aguas poco profundas, aguas protegidas del oleaje y aguas poco profundas y protegidas

Table 4. Relationship between percentages of manatee sightings on the southern coast of Puerto Rico vs. shallow waters, waters protected from the waves and shallow and protected waters

\begin{tabular}{lcccc}
\hline & $\begin{array}{l}\text { Aguas poco } \\
\text { profundas }\end{array}$ & $\begin{array}{l}\text { Aguas prote- } \\
\text { gidas }\end{array}$ & $\begin{array}{l}\text { Aguas poco profun- } \\
\text { das y protegidas }\end{array}$ & Otras áreas \\
\hline Avistamientos & $2.4 \%$ & $33.0 \%$ & $54.7 \%$ & $9.8 \%$ \\
$\begin{array}{l}\text { Pareja madre/ } \\
\text { cría }\end{array}$ & $0.9 \%$ & $38.1 \%$ & $51.3 \%$ & $9.7 \%$ \\
\multicolumn{1}{c}{$P$} & 0.0466 & 0.2339 & 0.0191 & 0.1960 \\
\hline
\end{tabular}

significativo para el predecir hábitat de manatíes $(P=0.0466)$ (Cuadro 4). La presencia de manatíes aumenta mientras menor es la profundidad y disminuye a mayor profundidad.

Se encontró que el grado de protección que proveen las bahías (al tomar en cuenta la profundidad, el oleaje y los vientos) es significativo para los avistamientos de manatíes. La mayoría de los avistamientos se dieron en aguas protegidas con oleaje menor a los $0.3 \mathrm{~m}$, ya sea en aguas poco profundas menores a los $3 \mathrm{~m}(54.7 \%)$ o mayores a los $3 \mathrm{~m}(33.0 \%)$ (Cuadro 4). Solo $9.8 \%$ de los avistamientos ocurrieron en áreas no protegidas, con oleaje mayor al $0.3 \mathrm{~m}$ y profundidades mayores a los $3 \mathrm{~m}$. Inclusive, manatíes fueron avistados solo en $2.4 \%$ de los avistamientos en aguas poco profundas (menores a los $3 \mathrm{~m}$ ), pero no protegidas y oleaje mayor a los $0.3 \mathrm{~m}$. Una relación similar se observó en los avistamientos de madres con cría y el grado de protección que proveen las bahías (aguas protegidas con oleaje menor a los $0.3 \mathrm{~m}$ y aguas poco profundas menores a los $3 \mathrm{~m}$ [51.3\%], o mayores a los $3 \mathrm{~m}$ [38.1\%], áreas no protegidas con oleaje mayor al $0.3 \mathrm{~m}$ y profundidades mayores a los $3 \mathrm{~m}$ [9.8\%], y aguas poco profundas menores a los $3 \mathrm{~m}$, pero no protegidas $\mathrm{y}$ oleaje mayor a los $0.3 \mathrm{~m}[0.9 \%]$ ).

\section{DISCUSIÓN}

Censos aéreos para manatíes son comúnmente llevados a cabo en la Florida (Reynolds et al. 2012), pero esporádicos, raros o ausentes para otros países dentro del rango de distribución de la especie. La mayoría de los censos de manatíes en estos países se llevaron a cabo con avionetas monomotor de ala alta (por ejemplo, 172 Skyhawk por Cessna Aircraft Company, Whichita, Kansas) (Reynolds et al. 2012), por el costo por hora de vuelo menor 
de la avioneta $v s$. el helicóptero. Mientras que las avionetas son más costo-efectivas para repetir censos aéreos, este estudio demuestra que el uso del helicóptero provee para mayor seguridad, y un conteo más certero del total de manatíes en un área. Esto se debe a que el helicóptero puede parar en sitio (planear estacionario), mientras se contabilizan todos los avistamientos y no hay que depender de volar en círculos para la contabilización detallada. Es durante estos vuelos en círculo cuando se pueden cometer errores de conteo doble o perder de vista a los manatíes, pero también es cuando la avioneta y la tripulación esta más expuesta a errores de aviación y accidentes (Lefebvre, 1995), especialmente si la avioneta es monomotor. Este estudio utilizó un helicóptero como plataforma de censo, lo que logró una mayor efectividad en detectar individuos y contabilizarlos.

Avistamientos y esfuerzo. El número promedio de manatíes avistado por censo aéreo durante este estudio (27.7, mínimo 5, máximo 69), es mayor al reportado para el área por Powell et al. (1981) (0-14), Rathbun et al. (1985) (0-10) y Freeman \& Quintero (1990) (0-8). El número de individuos avistados por hora de esfuerzo (promedio 13.0 individuos/h, mínimo 7.4 individuos/h, máximo 29.8 individuos/h) durante este estudio es también más alto que lo reportado para la misma área por Powell et al. (1981) (3.1), Rath- bun et al. (1985) (5.6) y Freeman \& Quintero (1990) (2.1). Este incremento es significativo al comparar el uso de avioneta con el de helicóptero como plataforma de avistamiento, con este último proveyendo mayor capacidad de avistamiento, conteo y reconteo de los manatíes. Más aún, su incremento en número por hora de esfuerzo desde el año 2007 al año 2015, representa un posible aumento poblacional, dada la tendencia clara de una pendiente positiva, forma de ver abundancia estandarizada para la especie (Fig. 4).

Agrupaciones. La agrupación por avistamiento varió entre dos y doce animales, para un promedio de 1.9 por grupo, similar al reportado por Powell et al. (1981) (1-6, promedio de 1.9), y es comparable a lo reportado para manatíes en México (1.8 en Morales-Vela et al. 2000, y 1.6 en Olivera-Gómez \& Mellink, 2002), pero más bajo de lo reportado para Panamá (2.2, Mou Sue et al. 1990). De los manatíes avistados, $12.4 \%$ fueron crías. En promedio, 3.5 parejas de madre/cría fueron observadas por censo aéreo en este estudio, con un promedio de 1.6 por horas de esfuerzo. El porcentaje de parejas de madre/ cría fue significativamente mayor que en los estudios anteriores, ambos para la isla entera $(6.4 \%$ en Powell et al. 1981 y $7.6 \%$ en Rathbun et al. 1985), y específicamente, para el área de estudio (3.4\% en Rathbun et al. 1985 y 10.3\% en Freeman \& Quintero, 1990). En Florida, $10 \%$ de avistamientos de parejas 
de madre/cría parece ser el promedio en censos aéreos (Packard \& Mulho1lend, 1983; Powell \& Rathbun, 1984), mientras que para México fue de $7.4 \%$ (Morales-Vela et al. 2000), 4.0\% para Honduras (Rathbun et al. 1983), 8.9\% y $10.6 \%$ para Belice (Bengston \& Magor, 1979; O’Shea \& Salisbury, 1991), y $15.7 \%$ para Panamá (Mou Sue et al. 1990). El alto porcentaje en crías en este estudio es de trascendencia, ya que indica la importancia del área para la crianza, específicamente los municipios de Salinas (44.7\%), seguido por Guánica $(27.3 \%)$ y Lajas $(25.8 \%)$.

Comportamiento. Los observados y su frecuencia variaron de los reportados por Rathbun et al. (1985). Sin embargo, esto se puede deber a diferencias en diseño de estudio e interpretación del observador. En adición, algunos comportamientos son inherentes a la naturaleza del manatí y no representan un caso particular significativo (por ejemplo, subiendo a la superficie a respirar, nadando, zambullendo). También, la interpretación de que un comportamiento es acertado (por ejemplo, descansando), es variable o no puede ser probado en su totalidad. Los que sin duda pueden verificarse son alimentación (si se nota remoción y flotación de hojas de yerbas marinas) y actividad reproductiva (cuando hay varios animales y demuestran comportamientos de copula o persecución de un individuo-presuntamente la hembra-en particular).
Por tanto, estudios futuros de censos aéreos no deberían enfocarse en una lista de comportamientos muy extensa y prestar más atención a los verificables, al tomar en cuenta la visión de Altmann (1974) de diferenciarlos entre estados y eventos.

Distribución temporal. En Puerto Rico se pueden observar manatíes todo el año a lo largo de la costa, con pequeñas variaciones por temporada, similar a lo observado por Powell et al. (1981), Rathbun et al. (1985) y Freeman \& Quintero (1990). La diferencia en el porcentaje de avistamiento, individuos y parejas de madre/cría, observada durante la época seca, puede deberse a mejores condiciones durante esta, dado a la poca precipitación y el poco flujo de agua marrón de los ríos, las cuales afectan la visibilidad. Ya que se da por hecho que los manatíes de Puerto Rico no inmigran o emigran a otras islas (Hunter $e t$ al. 2012), la leve variabilidad temporal puede ser más bien el resultado de las condiciones de visibilidad de agua para detectar manatíes.

Distribución espacial. La distribución de manatíes para la costa sur de Puerto Rico no es uniforme, con municipalidades que significativamente tienen mayor cantidad de avistamientos, animales y parejas de madre/cría. De particular uso se destacan Salinas, Guánica y Peñuelas para avistamiento $\mathrm{y}$ número de animales avistados, $\mathrm{y}$ Salinas, Guánica y Lajas para parejas de 
madre/cría. Los municipios con mayor porcentaje de avistamiento de grupos de reproducción fueron Guánica, Guayama y Peñuelas. Al tomar todos estos factores juntos, se despuntan entonces como áreas importantes para manatíes en la costa sur de Puerto Rico los mu- nicipios de Guánica (bahía de Guánica y ensenada las Pardas, Fig. 6B) y Lajas (bahía de la Parguera y bahía Montalva, Fig. 6A), Guayanilla (punta Verraco, Fig. 6C), Peñuelas (bahía de Tallaboa, Fig. 6C), Santa Isabel (punta Petrona, Fig. 7A), Salinas y Guayama (bahía de

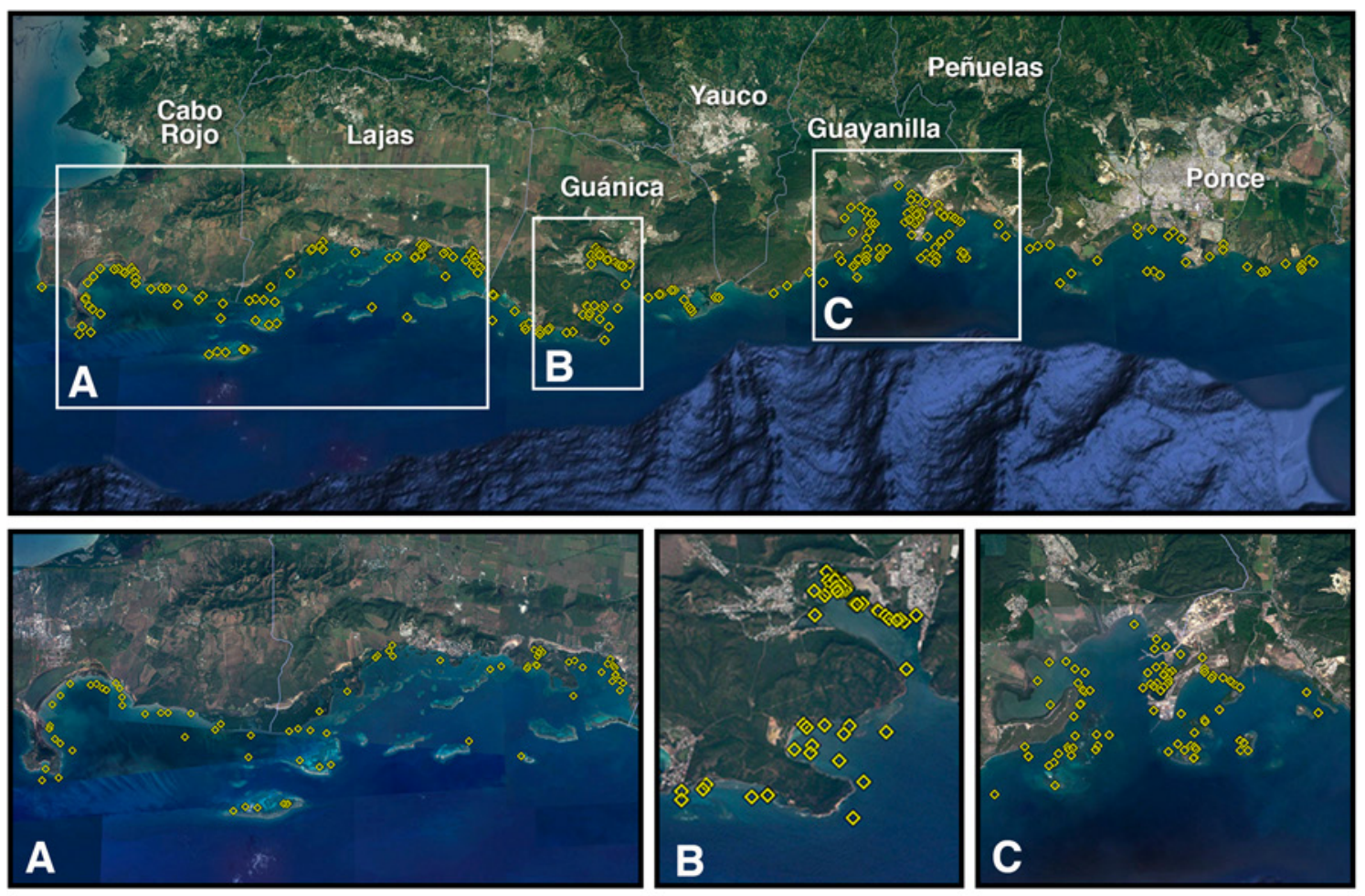

Fig. 6. Distribución de los avistamientos de manatíes para los censos aéreos llevados a cabo entre el 2001 y 2015 en la costa suroeste de Puerto Rico, se detallan las tres áreas de mayor uso de hábitat: A) bahía Sucia, La Parguera y cayos adyacentes en Cabo Rojo y Lajas, B) ensenada Las Pardas y bahía de Guánica y C) bahía de Guayanilla y bahía de Tallaboa en Guayanilla y Peñuelas

Fig. 6. Distribution of manatee sightings for aerial surveys conducted between 2001 and 2015 on the southwestern coast of Puerto Rico, detailing the three habitat areas of greatest use: A) bahía Sucia, La Parguera and adjacent cays in Cabo Rojo and Lajas, B) ensenada Las Pardas and bahía de Guánica, and C) bahía de Guayanilla and bahía de Tallaboa in Guayanilla and Peñuelas 
Jobos, Fig. 7B), y a menor grado, Cabo Rojo (bahía Sucia, Fig. 6A) y Arroyo y Peñuelas (punta Figuras, puerto de Patillas y bajo de Patillas, Fig. 7C), cónsono con lo detallado por Powell et al. 1981; Rathbun et al. 1985 y Drew et al. 2012. Sin embargo, el uso por manatíes de cayos lejanos a la costa en el sur de Puerto Rico y de la bahía Bioluminiscente reportado en este estudio no fue anteriormente documentado por Powell et al. 1981; Rathbun et al. 1985 o Freeman \& Quintero, 1990.

Zoogeográficamente, no se encontró que factores de temporada, temperatura superficial del mar o corrientes, afecten la presencia de manatíes. Tampoco, hubo diferencia significativa en relación con la cercanía de los manatíes a las yerbas marinas, inclusive si se considera a las parejas de madre/cría, aunque se entiende que es un factor limitante en la distribución de la especie (Lefebvre et al. 2000; Reid et al. 2001). No obstante, la relación no significativa en este estudio, la importancia de las praderas de yerbas marinas para la especie, es clara cuando se ve que en las grandes praderas en la costa de Cabo Rojo (bahía Sucia), Lajas (bahía Montalva) y Guayanilla (punta Verraco), siempre se observaron manatíes en todos los censos (Fig. 6A, 6C). Tanto, es así que Drew et al. (2012) sugirió que el área de Santa Isabel, entre punta Petrona y cayo Barbería (Fig. 7A), se considere como una región con un alto potencial para ser designada como área de protección del manatí, por su gran extensión de pradera de yerbas marinas.

Se ha detallado también que las fuentes de agua dulce son uno de los factores limitantes para los manatíes (Ortíz et al. 1998). Durante este estudio, fueron avistados tanto en la cercanía a la desembocadura de ríos en la costa sur de Puerto Rico (río Loco en Guánica, río Tallaboa en Peñuelas, río Coamo en Santa Isabel, río Seco en Guayama, río Nigua en Arroyo y río Grande de Patillas), inclusive dentro de dos de ellos (río Loco en Guánica y río Bucaná en Ponce). Sin embargo, el promedio de distancia a la fuente de agua dulce más cercana fue de $6.8 \mathrm{~km}$ para todos los avistamientos y $8.5 \mathrm{~km}$ para parejas de madre/cría. Debemos considerar que Puerto Rico es una isla kársica (Giusti, 1978), y la presencia de ríos submarinos que dan al mar y ojos de agua es alta, pero poco estudiada o documentada, las cuales podrían ser utilizadas por los manatíes como fuentes de agua dulce para su metabolismo y función renal. Estas fuentes de agua dulce son indetectables al ojo humano y por tanto no documentadas en este estudio.

Puerto Rico, específicamente la costa sur de la isla, presenta velocidades de corrientes promedio bajas, durante todo el año $(<10 \mathrm{~cm} / \mathrm{s})$ y esto crea un ambiente de baja energía cinética en el sitio, lo que hace que esta zona sea apropiada para los manatíes. La temperatura superficial del mar 

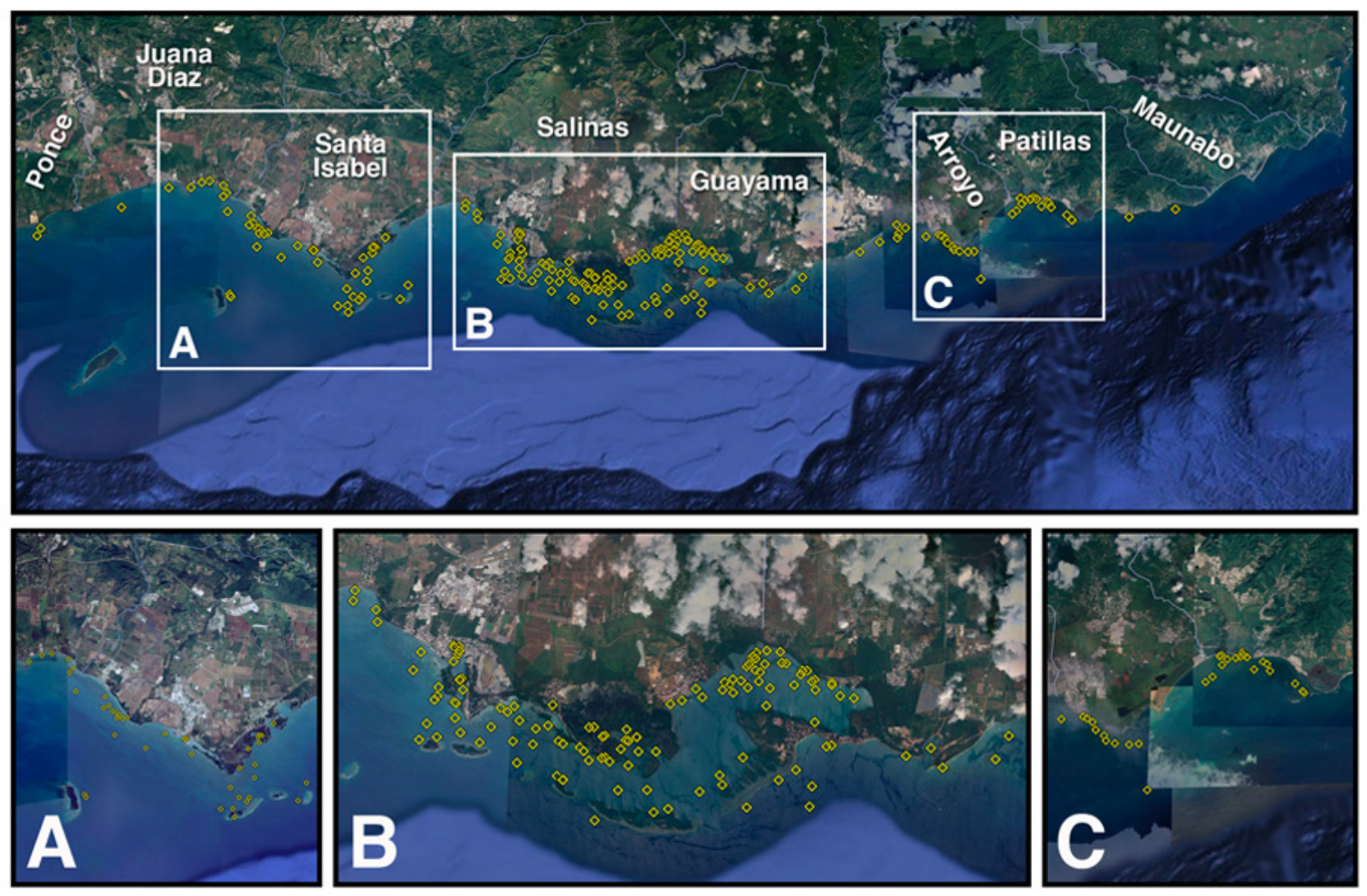

Fig. 7. Distribución de los avistamientos de manatíes para los censos aéreos llevados a cabo entre el 2001 y 2015 en la costa sureste de Puerto Rico, se detallan las tres áreas de mayor uso de hábitat: A) punta Petrona y bahías y cayos adyacentes en Santa Isabel, B) estuario de la bahía de Jobos en Salinas y Guayama, y C) punta Figuras, puerto de Patillas y bajo de Patillas

Fig. 7. Distribution of manatee sightings for aerial surveys conducted between 2001 and 2015 on the southeastern coast of Puerto Rico, detailing the three habitat areas of greatest use: A) punta Petrona and adjacent bays and cays in Santa Isabel, B) estuary of the bahía de Jobos in Salinas and Guayama, and C) punta Figuras, puerto de Patillas and bajo de Patillas

tampoco varía mucho durante el año, se mantienen entre los $27^{\circ} \mathrm{C}$ y $29^{\circ} \mathrm{C}$. Esto demuestra una baja influencia para la distribución de los manatíes en la isla, ya que no se registraron menores a $20^{\circ} \mathrm{C}$, y esto se considerada como limitante para que los manatíes busquen refugio durante el invierno en lugares donde el agua está por encima de los $22^{\circ} \mathrm{C}$ (Irvine \& Campbell, 1978). 
La profundidad ( $\mathrm{Z}$ ) del agua de los avistamientos mostró una mayor correlación con la distribución de los manatíes, tanto para los individuos como para las parejas de madre/cría. Esto demuestra que ellos prefieren aguas poco profundas (aproximadamente $3 \mathrm{~m}$ ), aunque en ocasiones se aventuran a aguas con mayor $\mathrm{Z}$, particularmente para moverse de un área a otra. Esto es de suma importancia para el manejo de la especie ante la amenaza por choques con embarcaciones a velocidad. Los deportes acuáticos, en particular carreras de bote y jet-ski, deben mantenerse lejos de estas aguas $(<$ $3.4 \mathrm{~m}$ de Z), preferidas y utilizadas por esta especie. A pesar de que los manatíes en Puerto Rico pasan mucho tiempo atravesando aguas costeras abiertas, prefieren aguas protegidas, como bahías poco profundas $(<3 \mathrm{~m}$ de $\mathrm{Z})$ con baja energía de oleaje $(<0.3 \mathrm{~m}$ de altura de la ola), para alimentarse, descansar y proteger a sus crías, similar a lo detallado por Drew et al. (2012).

\section{CONCLUSIÓN}

Dado que la supervivencia de los manatíes en Puerto Rico está amenazada por diferentes factores naturales, genéticos, por embotellamiento, falta de inmigración (Hunter et al. 2012), antrópicos, por caza directa y últimamente, por choques por embarcaciones a velocidad (Mignucci-Giannoni et al. 2000; Bonde et al. 2012), los datos, los mapas y los análisis producto de estos censos aéreos, junto a datos de telemetría y uso de hábitat (Reid \& Bonde, 1993; Reid et al. 1994a; Reid et al. 1994b; Deutsch et al. 1998, Slone et al. 2006, Reid et al. 2007), deben ayudar a delinear estrategias para designar áreas protegidas críticas, para el futuro de esta especie en peligro de extinción. El manatí antillano en Puerto Rico solo sobrevivirá cuando se consideren estudios multifacéticos (de genética, biología, etología, fisiología, patología, demografía y ecología) con acciones multisectoriales de conservación que incluyan ciencia, manejo y alcance comunitario.

\section{AGRADECIMIENTOS}

Este proyecto fue financiado por EcoEléctrica Puerto Rico y AES, especialmente gracias al apoyo de I. E. Laborde y Y. Alancastro, y se llevó a cabo bajo permisos del US Fish and Wildlife Service (MA791721-2, MA7917213, MA791721-4, MA791721-5, USGS Biological Resources Division Sirenia Project) y del Departamento de Recursos Naturales y Ambientales de Puerto Rico (DRNA-01-EPE-002, DRNA2014-EPE-27). Agradecemos la ayuda de observadores durante los vuelos, se incluye a A. R. Estrada-Acosta, C. I. Rivera-Pérez, G. M. Toyos-González, I. Cruz-Cruz, M. M. Colón-Llavina, M. 
A. Rodríguez-López, M. Pérez-Lewis, N. Landrau-Giovanetti, T. Alejando-Zayas y V. M. Rosado-Odom, y en especial la pericia de nuestros pilotos, J. L. Rodríguez-Pérez, J. A. Rodríguez-Rodríguez, P. Andino, A. Rodríguez y J. Cruz-Castro de Coptco Helicopters y Glide Aviation (Caribbean Heli-Jets). Agradecemos también a R. Villamil de Glide Aviation por todas las concesiones hechas para lograr el estudio a través de todos estos años; a D. Fernández por la asistencia con la estadística, y a J. P. Zegarra por la ayuda con los datos oceanográficos. Agradecemos los comentarios y las sugerencias de J. A. Powell, Bert Rivera-Marchand y evaluadores, en el manuscrito del estudio.

\section{BIBLIOGRAFÍA}

Altmann, J. (1974). Observational study of behavior: Sampling methods. Behaviour, 49(3-4), 227-267. https://doi. org/10.1163/156853974X00534.

Bengtson, J. L. \& Magor, D. (1979). A survey of manatees in Belize. J. Mammal, 60(1), 230-232. https://doi. org/10.2307/1379785.

Bonde, R. K., Mignucci-Giannoni, A. A. \& Bossart, G. D. (2012). Sirenian pathology and mortality assessment. En E. Hines, J. Reynolds, L. Aragones, A. A. Mignucci Giannoni \& M. Marmontel (Eds.), Sirenian Conservation: Issues and strategies in developing countries (pp. 148-156). Florida, EE.UU.: University Press of Florida.
Deutsch, C. J., Bonde, R. K. \& Reid, J. P. (1998). Radio-tracking manatees from land and space: Tag design, implementation, and lessons learned from long-term study. Mar. Technol. Soc. J., 32(1), 18-29.

Deutsch, C. J., Self-Sullivan, C. \& Mignucci-Giannoni, A. (2008). Trichechus manatus. The IUCN Red List of Threatened Species 2008: e. T22103A9356917. Recuperado en 1 de febrero de 2018, disponible en http:// dx.doi.org/10.2305/IUCN.UK.2008. RLTS.T22103A9356917.en.

Domning, D. P. \& Hayek, L. C. (1986). Interspecific and intraspecific morphological variation in manatees (Sirenia: Trichechus). Mar. Mamm. Sci., 2, 87-144. https://doi. org/10.1111/j.1748-7692.1986. tb00034.x.

Drew, C. A., Alexander-Vaughn, L. B. \& Collazo, J. A. (2012). Science summary in support of manatee protection area (MPA) design in Puerto Rico. North Carolina Agricultural Research Service, North Carolina State University, Tech. Bull., 330, 1-63. https://dx.doi. org/10.3996/CSS.101.2013.

Duarte, C. M., Marba, N. \& Santos, R. (2004). What may cause loss of seagrasses? En J. Borum, C. M. Duarte, D. Krause-Jensen \& T. M. Greve (Eds.), European seagrasses: An introduction to monitoring and management. (pp. 24-32). Hillerød, Denmark: M\&MS Project.

Freeman, J. \& Quintero, H. (1990). The distribution of West Indian manatees (Trichechus manatus) in Puerto Rico: 1988-1989. Nat. Tech. Info. Serv., PB91-137240, 1-43.

Giusti, E. V. (1978). Hydrogeology of the karst of Puerto Rico. U. S. Geological Survey Professional Paper 1012, 1-68. 
Google Earth. (2016). Google Earth ${ }$, Versión 7. Boston, EE.UU.: Free Software Foundation, Inc.

Hunter, M. E., Mignucci-Giannoni, A. A., Pause Tucker, K., King, T. L., Bonde, R. K., Gray, B. A. \& McGuire, P. M. (2012). Puerto Rico and Florida manatees represent genetically distinct groups. Conserv. Genet., 13, 16231635. https://doi.org/10.1007/s10592012-0414-2.

Irvine, A. B. \& Campbell, H. W. (1978). Aerial census of the West Indian manatee, Trichechus manatus, in the Southeastern United States. J. Mammal., 59, 613617. https://doi.org/10.2307/1380237.

Kendall, M. S., Monaco, M. E., Buja, K. R., Christensen, J. D., Kruer, C. R., Finkbeiner, M. \& Warner, R. A. (2001). Methods used to map the benthic habitats of Puerto Rico and the U.S. Virgin Islands. Maryland, EE.UU.: National Oceanic and Atmospheric Administration, National Ocean Service.

Lefebvre, L. W. (1995). Manatee aerial survey safety rules. SireNews, 24, 2-3.

Lefebvre, L. W. \& Kochman, H. I. (1991). An evaluation of aerial survey replicate count methodology to determine trends in manatee abundance. Wildl. Soc. Bull., 19, 298-309.

Lefebvre, L. W., Ackerman, B. B., Portier, K. M. \& Pollok, K. (1995). Aerial Survey as a technique for estimating trends in manatee population size-problems and prospects. En T. J. O'Shea, B. B. Ackerman \& H. R. Percival (Eds.), Population biology of the Florida manatee (pp. 63-74). Washington DC, EE.UU.: National Biological Service.

Lefebvre, L. W., Reid, J. P., Kenworthy, J. \& Powell, J. A. (2000). Characterizing manatee hábitat use and seagrass grazing in Florida and Puerto Rico: Implications for conservation and management. Pac.
Conserv. Biol., 5, 289-298. https://doi. org/10.1071/PC000289.

López-Marrero, T. \& Villanueva-Colón, N. (2006). Atlas ambiental de Puerto Rico. San Juan, Puerto Rico: La Editorial, Universidad de Puerto Rico.

Magor, D. (1979). Surey of the Caribbean manatee, Trichechus manatus L. on Vieques Island, Puerto Rico. Ceiba, Puerto Rico: Roosevelt Roads Naval Station, Department of the Navy.

Microsoft Excel. (2011). Microsoft Excel®, Versión 14.7.3. Boston, EE.UU.: Free Software Foundation, Inc.

Mignucci-Giannoni, A. A. (1989). Zoogeography of marine mammals in Puerto Rico and the Virgin Islands. Tésis de maestría no publicada, University of Rhode Island, Kingston.

Mignucci-Giannoni, A. A. \& Beck, C. A. (1998). The diet of the manatee (Trichechus manatus) in Puerto Rico. Mar. Mamm. Sci., 14(2), 394-397. https:// doi.org/10.1111/j.1748-7692.1998. tb00733. $\mathrm{x}$

Mignucci-Giannoni, A. A., Montoya-Ospina, R. A., Jiménez-Marrero, N. M., Rodríguez-López, M. A., Williams, E. H. \& Bonde, R. K. (2000). Manatee mortality in Puerto Rico. Environ. Manage., 25(2), 189-198. https://doi. org/10.1007/s002679910015.

Mignucci, A. (2010). El manatí de Puerto Rico. San Juan, Puerto Rico: Red Caribeña de Varamientos \& Universidad Interamericana de Puerto Rico.

Miller, K. E., Ackerman, B. B., Lefebvre, L. W. \& Clifton, K. B. (1998). An evaluation of strip-transect aerial survey methods for monitoring manatee populations in Florida. Wildl. Soc. Bull., 26, 561-570.

Morales-Vela, B., Olivera-Gómez, L. D., Reynolds, J. E. \& Rathbun, G. B. (2000). Distribution and habitat use by manatees 
(Trichechus manatus manatus) in Belize and Chetumal Bay, Mexico. Biol. Conserv., 95(1), 67-75. https://doi. org/10.1016/S0006-3207(00)00009-4.

Mou Sue, L. L., Chen, D. H., Bonde, R. K. \& O’Shea T. J. (1990). Distribution and status of manatees (Trichechus manatus) in Panama. Mar. Mamm. Sci., 6, 234-241. https:// doi.org/10.1111/j.1748-7692.1990. tb00247.x.

O’Shea, T. J. \& Salisbury, C. A. (1991). Belize-A last stronghold for manatees in the Caribbean. Oryx, 25, 156-164.

Olivera-Gómez, L. D. \& Mellink, E. (2002). Spatial and temporal variation in counts of the Antillean manatee (Trichechus manatus manatus) during distribution surveys at bahía de Chetumal, Mexico. Aquat. Mamm., 28, 285-293.

Ortíz, R. M., Worthy, G. A. J. \& MacKenzie, D. S. (1998). Osmoregulation in wild and captive West Indian manatees (Trichechus manatus). Physiol. Biochem. Zool., 71(4), 449-457. https://doi. org/10.1086/515427.

Packard, J. M. (1985). Development of manatee aerial survey techniques. Florida, EE.UU.: Cooperative Fish and Wildlife Research Unit, University of Florida.

Packard, J. M. \& Mulholland, R. (1983). Analysis of manatee aerial surveys: a compilation and preliminary analysis of winter aerial surveys conducted in Florida between 1977 and 1982. Florida, EE.UU.: Cooperative Fish and Wildlife Research Unit, University of Florida.

Packard, J. M., Siniff, D. B. \& Cornell, J. A. (1986). Use of replicate counts to improve indices of trends in manatee abundance. Wildl. Soc. Bull., 14, 265-275.

Packard, J. M., Summers, R. C. \& Barnes, L. B. (1985). Variation of visibility bias during aerial surveys of manatees. $J$.
Wildl. Manage., 49, 347-351. https:// doi.org/10.2307/3801528.

Powell, J. A. \& Rathbun, G. B. (1984). Distribution and abundance of manatees along the northern coast of the Gulf of Mexico. Northeast Gulf Sci., 7, 1-28.

Powell, J. A., Belitsky, D. W. \& Rathbun, G. B. (1981). Status of the West Indian manatee (Trichechus manatus) in Puerto Rico. J. Mammal., 62(3), 642-646. https://doi.org/10.2307/1380417.

Provancha, J. A. \& Provancha, M. J. (1988). Long-term trends in abundance and distribution of manatees (Trichechus manatus) in the northern Banana Rivera, Brevard County, Florida. Mar. Mamm. Sci., 4, 323-338. https://doi.org/10.1111/j.1748-7692.1988.tb00541.x

Rathbun, G. B. (1988). Fixed-wing airplane versus helicopter surveys of manatees (Trichechus manatus). Mar. Mamm. Sci., 4, 71-75. https://doi.org/10.1111/j.1748-7692.1988.tb00185.x.

Rathbun, G. B. \& Possardt, E. L. (1986). Recovery plan for the Puerto Rico population of the West Indian (Antillean) manatee (Trichechus manatus Linnaeus, 1758). Georgia, EE.UU.: US Fish Wildlife Service.

Rathbun, G. B., Carr, T., Carr, N. \& Woods, C. A. (1985). The distribution of manatees and sea turtles in Puerto Rico, with emphasis on Roosevelt Roads Naval Station. Nat. Tech. Info. Serv., PB86151834/GAR, 1-83.

Rathbun, G. B., Powell, J. A. \& Cruz, G. (1983). Status of the West Indian manatee in Honduras. Biol. Conserv., 26(4), 301-308. https://doi.org/10.1016/00063207(83)90094-0.

Reep, R. L., Bonde, R. K. (2010). The Florida manatee: Biology and conservation. Florida, EE.UU.: University of Florida Press. 
Reid, J. P. \& Bonde, R. K. (1993, noviembre). Can Puerto Rican manatees be tracked from space? The first satellite- based telemetry of Trichechus manatus outside the continental United States. En Abstract 10th Biennial Conference on the Biology of Marine Mammals, Galveston, Texas.

Reid, J. P., Bonde, R. K., Easton, D. E. \& Kochman, H. I. (1994a). 1993 annual report on the radio telemetry of manatees in Puerto Rico. Washington DC, EE.UU.: National Biological Service.

Reid, J. P., Bonde, R. K., Easton, D. E. \& Kochman, H. I. (1994b, marzo). Considerations for conducting manatee telemetry studies outside of Florida: A Puerto Rico case study. En Abstracts First International Manatee and Dugong Research Conference, Gainesville, Florida.

Reid, J. P., Easton, D. \& Kenworthy, W. J. (2001). Manatee and seagrass habitat characterization in Puerto Rico. Florida, EE.UU.: Roosevelt Roads Naval Station, Department of the Navy.

Reid, J., Jiménez, N., Butler, S., Montalvo, J., Slone, D., Saliva, J. \& Stith, B. (2007, noviembre). Use of Argos- linked GPS tags to document specific habitat use patterns of manatees in eastern Puerto Rico. En Abstracts 16th Biennial Conference on the Biology of Marine Mammals, Cape Town, South Africa.

Reynolds, J. E., Morales-Vela, B., Lawler, I. \& Edwards, H. (2012). Utility and design of aerial surveys for sirenians. En E. Hines, J. Reynolds, L. Aragones, A. A. Mignucci Giannoni \& M. Marmontel (Eds.), Sirenian Conservation: Issues and strategies in developing countries (pp. 186-195). Florida, EE.UU.: University Press of Florida.

Rstudio. (2016). Rstudio®. Versión 0.99.903. Boston, EE.UU.: RStudio Inc.

Self-Sullivan, C. \& Mignucci-Giannoni, A. A. (2008). Trichechus manatus ssp. manatus. The IUCN Red List of Threatened Species 2008: e.T22105A9359161. Recuperado en 1 de febrero de 2018, disponible en https://doi.org/10.2305/IUCN. UK.2008.RLTS.T22105A9359161.en.

Self-Sullivan, C. \& Mignucci-Giannoni, A. A. (2012). West Indian manatees (Trichechus manatus) in the Wider Caribbean Region. En E. Hines, J. Reynolds, L. Aragones, A. A. Mignucci Giannoni \& M. Marmontel (Eds.), Sirenian Conservation: Issues and strategies in developing countries (pp. 36-46). Florida, EE.UU.: University Press of Florida.

Slone, D. H., Reid, J. P., Bonde, R. K., Butler, S. M. \& Stith, B. M. (2006). Summary of West Indian manatee (Trichechus manatus) tracking by USGS-FISC Sirenia Project in Puerto Rico: With additional information on aerial surveys, carcass recovery and genetics research. Florida, EE.UU.: US Fish and Wildlife Service. 